\title{
A Clinicopathological Analysis of 120 Cases of Prostate Biopsies and Their Correlation with Prostate Specific Antigen levels
}

\author{
Anu Sumi Issac ${ }^{1 *}$, Ashley Ann Varughese ${ }^{1}$, C V Raghuveer ${ }^{2}$, Asha S P1, Preetha K ${ }^{1}$ \\ N Abdul Majeed ${ }^{1}$ and Junu Rajan ${ }^{3}$ \\ ${ }^{1}$ Department of Pathology, SUT Academy of Medical Sciences, Vencode PO, Vattapara, , Thiruvananthapuram - 695028, Kerala, India. \\ ${ }^{2}$ Department of Pathology Sri Devaraj Urs Academy of Higher Education and Research, Tamaka, Kolar-563103, Karnataka, India \\ ${ }^{3}$ Department of Pathology, Dr SMCSI Medical College, Parassala Vellarada Road, Thiruvananthapuram - 695504, Kerala, India
}

\begin{abstract}
Background: Interpretation of prostate lesions in TURP and needle biopsies have always been a challenge due to their varied presentation. PSA, produced by epithelial cells of all types of prostatic tissue, is the key factor for screening and detection of prostate cancer. The present study aimed to analyze the histopathological changes of prostate and correlate them with PSA levels.

Methods: One hundred and twenty cases of prostate lesions were obtained in two years. H\&E stained sections were examined and a histopathological diagnosis was given.

Result: Out of the 120 cases, 92 (76.7\%) were Benign Prostatic Hyperplasia (BPH), 9 (7.5\%) were Prostatic Intraepithelial Neoplasia (PIN) and $19(15.8 \%)$ were Prostate Carcinoma (PCa). All three lesions presented predominantly with obstructive lower urinary tract symptoms. In the BPH group, mean age was 66 years and mean PSA of $7.21 \mathrm{ng} / \mathrm{mL}$. In the PIN group, majority were low grade PIN (77.8\%), mean age of 66.56 years and mean PSA $38.23 \mathrm{ng} / \mathrm{mL}$. The highest PSA obtained in this study was $199 \mathrm{ng} / \mathrm{mL}$ in a case of low grade PIN. In the Prostate carcinoma group, mean age was 66 years, mean PSA $63.8 \mathrm{ng} / \mathrm{mL}$, majority were conventional adenocarcinomas (94.7 \%). Most of the carcinomas had a Gleason sum $<6$ and were well differentiated tumours $(52.6 \%)$.
\end{abstract}

Conclusion: All three groups had a common mean age of presentation and serum PSA levels were not always conclusive of the concerned pathology. Therefore, prostate lesions should be thoroughly evaluated by histopathology keeping in mind their neoplastic potential.

Keywords: Prostate, BPH, PIN, carcinoma, PSA, Gleason score

\section{Introduction}

Prostate and its associated lesions - their causes, biopsy sampling, its interpretation and PSA levels have been studied extensively. However, it is alarming to note that the second leading cause of death from cancer among men worldwide is prostate cancer and thus has evolved into an increasingly significant health problem. What adds fuel to the fire is the multifaceted presentation of benign or malignant or even potentially malignant lesions of prostate. Thus, a good command of benign lesions occurring in the prostate, and knowledge of their neoplastic potential will help pathologists confidently differentiate malignancy from the benign mimickers.

Many clinicians find prostatitis difficult to treat effectively. ${ }^{[1]} \mathrm{BPH}$ is observed as a strictly age-related phenomenon in nearly all men, starting at approximately 40 years of age. PIN may display a spectrum of architectural patterns, from a simple flat epithelium to a complex cribriform pattern that may be difficult to distinguish from cribriform carcinoma. ${ }^{[2]}$ Prostate cancers are slow growing, less symptomatic, remain unrecognized clinically and have an unpredictable course in its speed of progression. ${ }^{[1]}$ PSA, secreted by both benign and malignant prostatic tissue, is more sensitive than PAP (Prostatic Acid Phosphatase) in the detection of prostate carcinoma. ${ }^{[3]}$ With this background, the present study aimed to understand the incidence, clinical presentation of prostatic lesions (non-neoplastic and neoplastic), its different histopathological patterns including rare variants, their grading and correlation with PSA levels.

\section{Materials and Methods:}

This is a study of 120 cases of prostate biopsy received in the Department of Pathology in a private medical college hospital over a period of two years. The institutional review board approval was obtained prior to the beginning of the study. The clinical presentation, history, PSA levels and other relevant findings were obtained from patient records available in the department.

The specimens received in 10\% formalin consisted of either TURP, Needle biopsies or Radical Prostatectomy. All the material was processed by paraffin embedding technique and 4-micron thick sections obtained were stained with H\&E. For ease of analysis, the samples were subdivided into 3 groups - BPH, PIN and PCa group. 


\section{Result}

Of the 120 prostate biopsies, we diagnosed 92 (76.6\%) BPH, 9 (7.5\%) PIN and 19 (15.8\%) PCa. Majority were obtained through TURP (96 cases, 80\%), 23 needle biopsies (19.2\%) and 1 radical prostatectomy $(0.83 \%)$.

We observed that majority of BPH and PIN were found between $6^{\text {th }}$ and $7^{\text {th }}$ decades while carcinoma was predominant in the $5^{\text {th }}$ decade with 55 years being the youngest age at presentation. All 3 groups showed a common mean age of presentation - 66 years. (Table 1)

Obstructive urinary symptoms predominated in all 3 groups with difficulty in micturition being the most common presentation. In BPH group, majority of the patients had PSA $\leq 10 \mathrm{ng} / \mathrm{mL}$. In this study, the highest PSA obtained was $199 \mathrm{ng} / \mathrm{mL}$ observed in a LGPIN patient. The highest mean PSA level was in the PCa group. (Table 2)

Microscopically BPH was diagnosed based on glandular as well as fibromuscular stromal hyperplasia. In glandular hyperplasia, the glands varied in size from medium to large, some of them were cystically dilated. The lining epithelium was bi-layered with inner tall columnar mucus secreting epithelium and outer cuboidal to flattened epithelium with basal nuclei. (Figure 1) Other histologic features included, basal cell hyperplasia seen in 13/92 (14.1\%) and squamous metaplasia seen in 3/92 (3.2\%). An inflammatory reaction of acute and chronic type was seen in 63/92 cases (68.5\%).

In low grade PIN, microscopically, the glandular architecture showed epithelial cell crowding and stratification with irregular spacing, enlarged nuclei with normal chromatin, inconspicuous nucleoli with intact basal cell layer and basement membrane. In high grade PIN, there was increased epithelial cell crowding and stratification with some lesions showing tufting, micropapillary, cribriform and flat patterns. The nuclei of the cells were enlarged and pleomorphic, chromatin was dense and clumped with prominent nucleoli. Basal cell layer and basement membrane were intact. (Figure 2) Three lesions of PIN were seen to be associated with basal cell hyperplasia and urothelial metaplasia. Chronic prostatitis was seen to be associated with $5 / 9(55.5 \%)$ cases

In PCa group, majority were conventional adenocarcinomas (94.7 \%) (Figure 3 ) and one case of ductal adenocarcinoma was obtained $(5.3 \%)$. The most common primary pattern was score $3(52.6 \%)$ and the most common secondary pattern was score $4(42.1 \%)$. The most common Gleason Sum were Score 6 and Score 7 (26.3\%). The ductal adenocarcinoma had a Gleason Sum of $6[3+3]$.

Based on the Gleason sum (GS), the prostate carcinomas can be graded into well differentiated (GS $<6$ ), moderately differentiated (GS 7) and poorly differentiated tumours (GS 8-10). In the present study, $10(52.6 \%)$ had GS $<6,5$ $(26.3 \%)$ had GS 7 and $4(21.1 \%)$ had GS 8-10.

With respect to the age specific reference range of PSA, in the category of 40-49 years, the mean PSA levels in BPH group was normal. However, all three groups produced high levels of PSA in 50-90 years age group much beyond the age-specific reference range for PSA. In the PCa group, all the lesions produced very high PSA, however, the mean PSA levels were found to be decreasing as age advanced.

Table 1: Age at presentation and Mean age of BPH, PIN and PCa groups.

\begin{tabular}{|c|c|c|c|c|c|c|}
\hline \multirow{2}{*}{$\begin{array}{l}\text { Age group } \\
\text { (years) }\end{array}$} & \multicolumn{2}{|c|}{ BPH } & \multicolumn{2}{|c|}{ PIN } & \multicolumn{2}{|c|}{$\mathrm{PCa}$} \\
\hline & No: of cases & Percentage $\%$ & No: of cases & Percentage $\%$ & No: of cases & Percentage \% \\
\hline $40-49$ & 2 & 2.17 & 0 & 0 & 0 & 0 \\
\hline $50-59$ & 12 & 13.04 & 2 & 22.2 & 6 & 31.6 \\
\hline $60-69$ & 48 & 52.17 & 3 & 33.3 & 6 & 31.6 \\
\hline $70-79$ & 26 & 28.26 & 3 & 33.3 & 4 & 21.1 \\
\hline $80-89$ & 4 & 4.35 & 1 & 11.2 & 3 & 15.7 \\
\hline TOTAL & 92 & 100 & 9 & 100 & 19 & 100 \\
\hline MEAN AGE & \multicolumn{2}{|c|}{66 years } & \multicolumn{2}{|c|}{66.56 years } & \multicolumn{2}{|c|}{66 years } \\
\hline
\end{tabular}

Table 2: PSA levels and Mean PSA of BPH, PIN and PCa groups.

\begin{tabular}{|c|c|c|c|c|c|c|}
\hline \multirow{2}{*}{ PSA levels (ng/mL) } & \multicolumn{2}{|c|}{ BPH } & \multicolumn{2}{|c|}{ PIN } & \multicolumn{2}{|c|}{$\mathrm{PCa}$} \\
\hline & No: of cases & Percentage $\%$ & No: of cases & Percentage \% & No: of cases & Percentage $\%$ \\
\hline $0-10$ & 73 & 79.35 & 1 & 11.1 & 1 & 5.26 \\
\hline $10-20$ & 9 & 9.78 & 2 & 22.2 & 3 & 15.79 \\
\hline $20-30$ & 6 & 6.52 & 5 & 55.6 & 5 & 26.32 \\
\hline $30-40$ & 3 & 3.26 & 0 & 0 & 0 & 0 \\
\hline $40-50$ & 0 & 0 & 0 & 0 & 2 & 10.53 \\
\hline
\end{tabular}


PSA Levels in Various Histological Types of Prostatomegaly

\begin{tabular}{|c|c|c|c|c|c|c|}
\hline \multirow{2}{*}{ PSA levels (ng/mL) } & \multicolumn{2}{|c|}{ BPH } & \multicolumn{2}{|c|}{ PIN } & \multicolumn{2}{|c|}{$\mathrm{PCa}$} \\
\hline & No: of cases & Percentage $\%$ & No: of cases & Percentage \% & No: of cases & Percentage \% \\
\hline $50-60$ & 0 & 0 & 0 & 0 & 0 & 0 \\
\hline $60-70$ & 1 & 1.08 & 0 & 0 & 1 & 5.26 \\
\hline $70-80$ & 0 & 0 & 0 & 0 & 2 & 10.53 \\
\hline $80-90$ & 0 & 0 & 0 & 0 & 0 & 0 \\
\hline $90-100$ & 0 & 0 & 0 & 0 & 0 & 0 \\
\hline $100-200$ & 0 & 0 & 1 & 11.1 & 5 & 26.31 \\
\hline TOTAL & 92 & 100 & 9 & 100 & 19 & 100 \\
\hline MEAN PSA & \multicolumn{2}{|c|}{$7.21 \mathrm{ng} / \mathrm{mL}$} & \multicolumn{2}{|c|}{$38.23 \mathrm{ng} / \mathrm{mL}$} & \multicolumn{2}{|c|}{$63.8 \mathrm{ng} / \mathrm{mL}$} \\
\hline
\end{tabular}

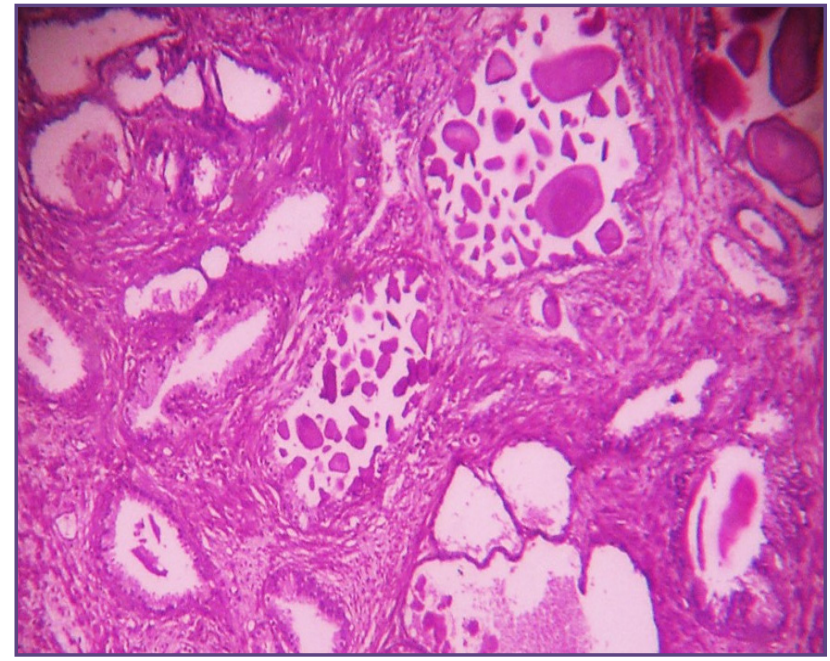

Fig. 1: Photomicrograph of BPH showing cystically dilated glands filled with inspissated secretions and corpora amylacea $(\mathrm{H} \& \mathrm{E}, 4 \mathrm{x})$.

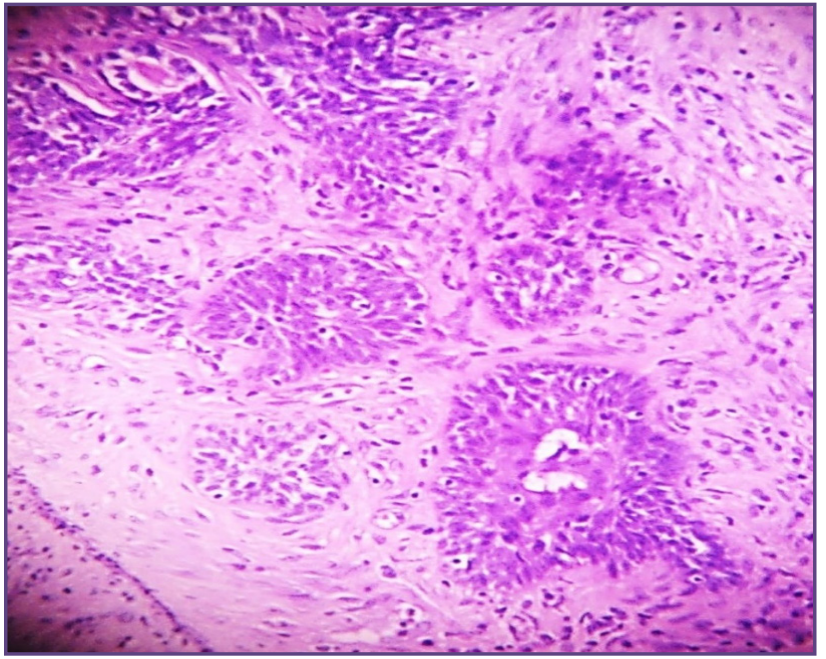

Fig. 2: Photomicrograph of High grade PIN showing smaller, crowded glands with stratification of epithelium, hyperchromatic nuclei (H\&E, 10x).

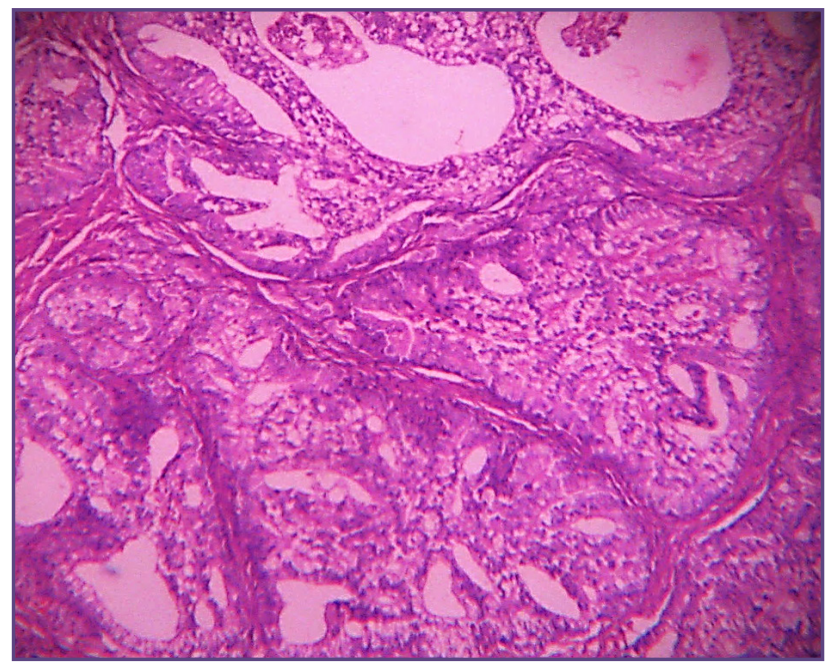

Fig. 3: Photomicrograph of Prostate Adenocarcinoma, Gleason Sum 9 (4+5) [H\&E, 4x]: Sharply circumscribed, rounded masses of cribriform glands with central necrosis lined by single layers of cuboidal cells, pleomorphic hyperchromatic nuclei and prominent nucleoli. 


\section{Discussion}

Among the benign proliferative lesions of prostate, benign prostatic hyperplasia was predominant. The patients were between 48-87 years of age and mean age of occurrence was 66 years. Majority of cases was found in the $7^{\text {th }}$ decade of life which was comparable to the study by Shirish et al. [4]

The most common manifestation of BPH is lower urinary tract symptom subdivided into obstructive and irritative symptoms ${ }^{[5]}$ In the present study, majority of the patients (79 cases, $85.87 \%$ ) presented with obstructive urinary symptoms with difficulty in micturition being the most

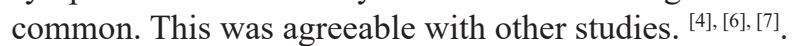

Benign prostatic tissue has been known to secrete PSAand their levels have been found to increase in non-malignant lesions of prostate such as BPH ${ }^{[3]}$. In the present study, the range of PSA observed in BPH was between 0.04-64.6ng/ $\mathrm{mL}$ and the mean PSA was 7.21 which is comparable with Lee et al. ${ }^{[8]}$ The probable reason for such a wide range in PSA could be due to the association with prostatitis which can raise PSA levels. Also, 19 cases (20.6\%) had PSA levels $>10 \mathrm{ng} / \mathrm{mL}$ which is higher compared to the study done by Lakhey et al ${ }^{[9]}$ where it was only 3 cases $(3.28 \%)$.

Amongst 9 cases of PIN, 2 cases (22.2\%) were high grade. The incidence and extent of PIN appears to increase with patient age. Most foci of PIN in young men are low grade, with increasing frequency of PIN with advancing age. ${ }^{[10]}$ In our study, the age range varied from 53-80 years, mean age was 66.56 years and majority patients were between the $7^{\text {th }}$ and $8^{\text {th }}$ decade of life, including HGPIN. This was in contrast to studies by Bostwick et al ${ }^{[11]}$ and Chapado et al ${ }^{\left[{ }^{[12]}\right.}$ where the highest incidence was in the $9^{\text {th }}$ decade.

Serum PSA concentrations may be elevated in patients with PIN. ${ }^{[13]}$ The highest PSA of $199 \mathrm{ng} / \mathrm{mL}$ was surprisingly found in a 64-year-old patient with LG PIN and prostatitis. The probable reason for such an alarming increase in PSA in a LGPIN could be attributed to the presence of prostatitis and possible repeated digital rectal examination for suspicion of carcinoma. ${ }^{[14]}$

Prostate cancer is rare before 40 years of age, but the incidence rises quickly thereafter. ${ }^{[1]} \mathrm{A}$ recent global prostate cancer evaluation found Indian men to have a high probability of advanced stage disease. ${ }^{[15]}$ In the present study, the youngest patient was 55 years and the most common age group affected was 50-60 years. A Nigerian study ${ }^{[16]}$ observed the most common occurrence between $60-70$ years while an Iranian study ${ }^{[17]}$ observed predominance in $70-80$ age group. We had majority of cases between 50-69 years which was comparable with the Nigerian study. ${ }^{[16]}$ Interestingly, although prostate cancer has been known as a disease of elderly men, studies have shown that mean age of patients at presentation with prostate cancer is decreasing. ${ }^{[18,19]}$ In the present study, the mean age of presentation was 66 years which was comparable to studies by Zhu et al ${ }^{[20]}$ and Mirzaie et al ${ }^{[21]}$.

Prostate cancer exhibits no specific presenting symptoms and is usually clinically silent, although it may cause urinary obstructive symptoms mimicking BPH. ${ }^{[1]}$ In the present study, obstructive urinary symptoms were most common and hence comparable to studies by Sapira et al ${ }^{[19]}$ and Oranusi et al ${ }^{[22]}$.

Cancerous prostate tissue usually releases more PSA into the blood than normal, healthy tissue. ${ }^{[23]}$ Numerous studies have shown that patients with prostate cancer have elevated serum PSA levels relative to men without prostate cancer. The most commonly used cut-off for PSA is $4 \mathrm{ng} /$ $\mathrm{mL} .{ }^{[24]}$ In the present study, the PSA range in PCa group was between $9.75-180 \mathrm{ng} / \mathrm{mL}$ and the mean PSA was 63.8 which is higher compared to the mean PSA level observed in studies by Ojewola et al ${ }^{[16]}$ and Zivkovic ${ }^{[25]}$. Also, it was observed in our study that majority of the cases had a PSA level $>10 \mathrm{ng} / \mathrm{mL}$ which was comparable to studies by Lakhey et al ${ }^{[9]}$ and Zivkovic ${ }^{[25]}$.

In the present study, the most common primary pattern in Prostatic Carcinoma was score $3(52.6 \%)$ and the most common secondary pattern was score 4 (42.1\%) which was similar to the study by Shirish et al. ${ }^{[4]}$

Divrik et al ${ }^{[26]}$ in their study had observed that Gleason sums of 6 and 7 were the most common scores which was comparable to the present study but all these specimens were needle biopsies. We had $50 \%$ cases each of TURP specimens and needle biopsies in the Gleason sum of 6 and 7. The single specimen obtained by radical prostatectomy in this study had a Gleason score of 10.

Gleason grading of prostate cancer remains as one of the most powerful factors predicting its nature and prognosis. ${ }^{[27]}$ In the present study, most of the carcinomas had a Gleason sum $<6$ and were well differentiated tumours $(52.6 \%)$. However, Shirish et al ${ }^{[4]}$ and Khang et al ${ }^{[28]}$ observed a predominance of moderately differentiated tumours with Gleason sum 7 with 9 cases (52.94\%) and 148 cases $(60.4 \%)$ respectively. We had 4 cases $(21.1 \%)$ falling in the poorly differentiated group with Gleason Sum $8-10$ which was comparable with Shirish et al ${ }^{[4]}$ (5 cases, $29.4 \%)$.

Ductal adenocarcinomas account for $<1 \%$ of prostate adenocarcinoma and are often seen in transurethral resection specimens. ${ }^{[29]}$ We had a single case $(5.3 \%)$ of 
ductal adenocarcinoma of prostate from a 65 -year-old male identified on needle biopsy with high PSA of $120 \mathrm{ng} / \mathrm{mL}$ and Gleason sum 6. Microscopy showed a papillary pattern of arrangement with glands lined by tall, pseudostratified epithelium with large hyperchromatic nuclei, mitosis and comedonecrosis. Tavora et al ${ }^{[30]}$ found 28 cases of prostate ductal adenocarcinoma between $50-91$ years with a mean age of 68 years and a PSA range between $1.2-12.1 \mathrm{ng} / \mathrm{mL}$. Our patient was diagnosed on needle biopsy and had high PSA levels which can be attributed to the comedonecrosis.

\section{Conclusion}

With advancements in modern medicine, we have become increasingly aware of potential risks of prostate pathologies. This may be the reason for earlier detection of an increasing number of PIN and PCa cases. Though age specific PSA levels and cut off PSA levels for malignancy have been put forth, we have found that PSA levels tend to vary considerably in different lesions and hence need not always be of diagnostic significance. Thus, benign lesions of prostate, their neoplastic potential and mimickers of malignancy deserve a thorough firsthand histopathological study so as to confidently diagnose them as well as improve therapy and follow-up for the patient.

\section{Abbreviations \\ BPH - Benign Prostatic Hyperplasia \\ TURP - Transurethral Resection of Prostate \\ PIN - Prostatic Intraepithelial Neoplasia}

LGPIN - Low Grade Prostatic Intraepithelial Neoplasia

HGPIN - High Grade Prostatic Intraepithelial Neoplasia

$\mathrm{PCa}$ - Prostatic carcinoma

GS - Gleason Sum

PSA - Prostate Specific Antigen

\section{Acknowledgements}

I wholeheartedly thank the laboratory technicians and medical records department staff for their cooperation and help in retrieval of patient data. I thank Dr C V Raghuveer for being my solid support and critic throughout the study. I would also like to thank my HOD Dr Preetha for helping me with the study.

\section{Reference:}

1. Bostwick DG, Amin MB. Prostate and seminal vesicles. In: Damjanov I, Linder J, editors. Anderson's Pathology. Diseases of the urogenital and reproductive systems. 2. 10th ed 2006. p. $2197-230$.

2. Ayala AG, Ro JY. Prostatic intraepithelial neoplasia Recent advances. Arch Pathol lab Med. 2007;131.
3. Epstein JI, Netto GJ. Clinical correlates with biopsy: Serum prostate-specific antigen, digital rectal examination, and imaging techniques. In: Epstein J I, Netto G J (eds) : Biopsy interpretation of the prostate. Philadelphia, Lippincott Williams and wilkins, 2007 pp 2-5.

4. Shirish C, Jadhav PS, Anwekar SC, Kumar H, Buch AC, Chaudhari US. Clinico-pathological study of benign \& malignant lesions of prostate. IJPBS. 2013;3(1):162-78.

5. Roehrborn CG. Benign prostatic hyperplasia : An overview. Reviews in Urology. 2005;7.

6. Herawi M, Epstein J. Specialised stromal tumors of the prostate: A clinicopathologic study of 50 cases. Am J Surg Pathol 2006;30(6):694-704.

7. Khan M, Khan A, Khan S, Nawaz H. Benign prostatic hyperplasia: mode of presentation and postoperative outcome. J Pak Med Assoc. 2005;55(1):20-3.

8. Lee JG, Bae SH, Choi SH, Kwon TG, Kim T-H. Role of Prostate-Specific Antigen Change Ratio at Initial Biopsy as a Novel Decision-Making Marker for repeat Prostate Biopsy. Korean J Urol. 2012;53:467.

9. Lakhey M, Ghimire R, Shrestha R, Bhatta A. Correlation of serum free prostate-specific antigen level with histological findings in patients with prostatic disease. Kathmandu University Medical Journal 2010;8(30):158-63.

10. Bostwick DG, Cheng L. Precursors of prostate cancer. Histopathology. 2012;60:4-27.

11. Bostwick DG, Qian J. High grade prostatic intraepithelial neoplasia. Modern Pathology 2004; 7:360-79.

12. Sánchez-Chapado M, Olmedilla G, Cabeza M, Donat E, Ruiz A. Prevalence of prostate cancer and prostatic intraepithelial neoplasia in Caucasian Mediterranean males: An autopsy study. The Prostate. 2003;54(3):238-47.

13. Bostwick DG, Pacelli A, Lopez-Beltran A. Molecular biology of prostatic intraepithelial neoplasia. The Prostate. 1996; 29:117-34.

14. Epstein JI, Netto GJ. Clinical correlates with biopsy: Serum prostate-specific antigen, digital rectal examination, and imaging techniques. In: Epstein J I, Netto G J (eds) : Biopsy interpretation of the prostate. Philadelphia, Lippincott Williams and wilkins, 2007 pp 2-5.

15. Zeigler-Johnson CM, Rennert H, Mittal RD, Jallo M, Sachdeva R, Malkowicz SB, et al. Evaluation of prostate cancer characteristics in four populations worldwide. Can J Urol. 2008;15(3):4056-64.

16. Ojewola R, TIjanu K, Jeje E, Anunobi C, Ogunjimi M, Ezenwa E, et al. Is extended biopsy protocol justified in all patients with suspected prostate cancer?. Nigerian Journal of Clinical Practice 2012;15(3):315-9.

17. Pourmand G, Allameh F, Mohammed K, Dehghani S, Pourmand B, Mehrsai A, et al. Prostate cancer predicting factors: A Preliminary report from Tehran. Urology Journal. 2012;9(4):667-72. 
18. Hsing AW, Sakoda LC, Jr SCC. Obesity, metabolic syndrome and prostate cancer. Am J Clin Nutr 2007;86:843S-8457S.

19. Sapira MK, Onwuchekwa AC, Onwuchekwa CR. Comorbid medical conditions and medical complications of Prostate Cancer in Southern Nigeria. Med J Malaysia. 2012;67(4):412-6.

20. Zhu Y-P, Ye D-W, Yao X-D, Zhang S-L, Dai B, Zhang H-L, et al. Prevalence of incidental prostate cancer in patients undergoing radical cystoprostatetectomy: data from China and other Asian countries. Asian Journal of Andrology. 2009;11:104-8.

21. Zare-Mirzaie A, Balvayeh P, Imamhadi MA, Lotfi M. The frequency of latent prostate carcinoma in autopsies of over 50 years old males, the Iranian experience. Medical Journal of Islamic Republic of Iran. 2012;26(2):73-7.

22. Oranusi C, Ugezu A, Nwofor A. Diagnosis of prostate cancer with needle biopsy: Should all cases be biopsied before treatment? Nigerian Journal of Clinical Practice 2012;15(1):48-50.

23. Arneth BM. Clinical significance of measuring prostatespecific antigen. 2009; 40: [487-91 pp.]. Available from: labmed.ascpjournals.org/content/40/8/487.full.

24. Epstein JI, Netto GJ. Clinical Correlates with Biopsy: Serum Prostate Specific Antigen, Digital rectal examination and
Imaging techniques. Biopsy Interpretation of the Prostate. 4th ed. Baltimoore: Lippincott Williams and Wilkins; 2008. p. $2-5$.

25. Zivkovic S. Correlation between prostate-specific antigen and histopathological difference of prostate carcinoma. Arch Oncol 2004;12(3):148-51.

26. Divrik RT, Eroglu A, Sahin A, Zorlu F, Ozen H. Increasing the number of biopsies increases the concordance of Gleason scores of needle biopsies and prostatectomy specimens. Urologic Oncology: Seminars and Original Investigations 2007;25:376-82.

27. Osunkoya AO. Update on Prostate Pathology. Pathology. 2012;44(5):391-406.

28. Khang IH, Kim YB, Yang SO, Lee JK, Jung TY. Differences in Postoperative Pathological Outcomes between Prostate Cancers Diagnosed at Initial and Repeat biopsy. Korean J Urol. 2012;53:531-5.

29. Grignon DJ. Unusual subtypes of prostate cancer. Modern Pathology. 2004;17:316-27.

30. Tavora F, Epstein JI. High grade prostatic intraepithelial neoplasia like ductal adenocarcinoma of the prostate: a clinicopathologic study of 28 cases. Am J Surg Pathol 2008;32(7):1060-7.

*Corresponding author:

Dr. Anu Sumi Issac, MBBS, MD, Panicker House, Arra 153 A, Tc 16/4230, Aishwarya Gardens, Kalakaumudi Road, Medical College P. O., Thiruvananthapuram - 695011, Kerala, India.

Phone: +91 8281605701

Email: dranuissac@gmail.com

Financial or other Competing Interests: None. 